\section{(6) OPEN ACCESS}

\title{
Profilin1 E117G is a moderate risk factor for amyotrophic lateral sclerosis
}

\author{
Pietro Fratta, ${ }^{1,2}$ James Charnock, ${ }^{2}$ Toby Collins, ${ }^{2}$ Anny Devoy, ${ }^{2}$ Robin Howard, ${ }^{1,3}$ \\ Andrea Malaspina, ${ }^{4}$ Richard Orrell, ${ }^{1,5}$ Katie Sidle, ${ }^{1,3}$ Jan Clarke, ${ }^{1,3}$ Maryam Shoai, ${ }^{5}$ \\ Ching-hua Lu, ${ }^{4,6}$ John Hardy, ${ }^{5}$ Vincent Plagnol, ${ }^{7}$ Elizabeth M C Fisher ${ }^{1,2}$
}

For numbered affiliations see end of article.

\section{Correspondence to}

Dr Pietro Fratta, Department of Neurodegenerative Diseases,

UCL Institute of Neurology, Queen Square, London WC1N 3BG, UK; p.fratta@prion.ucl. ac.uk

PF and JC contributed equally.

Received 10 September 2013 Revised 21 October 2013 Accepted 28 October 2013 Published Online First 5 December 2013

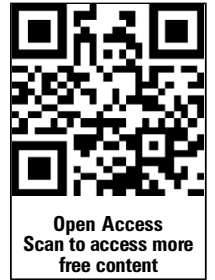

To cite: Fratta $P$, Charnock J, Collins T, et al. J Neurol Neurosurg Psychiatry 2014;85:506-508.

\section{ABSTRACT}

Background Amyotrophic lateral sclerosis (ALS) and frontotemporal dementia (FTD) are progressive neurodegenerative disorders that share significant clinical, pathological and genetic overlap and are considered to represent different ends of a common disease spectrum. Mutations in Profilin1 have recently been described as a rare cause of familial ALS. The PFN1 E117G missense variant has been described in familial and sporadic cases, and also found in controls, casting doubt on its pathogenicity. Interpretation of such variants represents a significant clinical-genetics challenge.

Objective and results Here, we combine a screen of a new cohort of $383 \mathrm{ALS}$ patients with multiple-sequence datasets to refine estimates of the ALS and FTD risk associated with PFN1 E117G. Together, our cohorts add up to $5118 \mathrm{ALS}$ and FTD cases and 13089 controls. We estimate a frequency of E117G of $0.11 \%$ in controls and $0.25 \%$ in cases. Estimated odds after population stratification is $2.44(95 \% \mathrm{Cl} 1.048$ to $\infty$, MantelHaenszel test $p=0.036$ ).

Conclusions Our results show an association between E117G and ALS, with a moderate effect size.

\section{INTRODUCTION}

Amyotrophic lateral sclerosis (ALS) is a devastating neurodegenerative disorder in which the progressive loss of motor neurons causes weakness and paralysis leading to death typically $3-5$ years after onset. ${ }^{1}$ Around $5-10 \%$ of ALS cases are familial, which has allowed the identification of a handful of mutant genes that cause classical dominant adult-onset ALS, and are occasionally found in sporadic patients with ALS, who report no family history for the disease. ${ }^{2}$ Frontotemporal dementia (FTD) co-occurs in $5-10 \%$ of patients with ALS and the finding that a number of mutations can cause either disorder, and that ALS and FTD postmortem pathology observations share numerous similarities, has led to these disorders being considered part of the same disease spectrum. ${ }^{3}$

Recently, Wu et $a l^{4}$ have identified missense mutations in Profilin 1 (PFN1), a three-exon gene encoding a protein involved in actin filament formation, as causative of ALS. With the exception of the E117G mutation, all mutant PFN1 alleles were found in familial ALS cases. One successive study identified a novel missense mutation, while a number of screening studies have failed to identify mutations, suggesting these are a rare cause for
ALS. By contrast, the E117G mutation was identified in control and familial cases, and was also found at a lower frequency in controls in other populations, thus casting a doubt on the significance of this variant. ${ }^{5-11}$

Here we report the screening of a UK population for PFN1 mutations. We identify cases carrying the E117G variant and analyse the frequency of this variant in $4700 \mathrm{UK}$ controls. We then meta-analyse all the published and publicly available data for this variant, to conclude this may represent a rare risk factor for ALS.

\section{METHODS}

Study population

Three hundred and eighty-three sporadic and familial ALS cases, from specialist motor neuron disease clinics in London, UK, (UCL Partners motor neuron disease clinics), provided written informed consent. The familial cases were 23 and represent $6 \%$ of the cohort. DNA was extracted from whole blood.

\section{Sequencing analysis}

PFN1 exons 1, 2 and 3 were subjected to PCR amplification by REDtaq Polymerase (Sigma), using the primers and PCR conditions as described in Wu et al. ${ }^{4}$ Reaction mixtures were made up in accordance with the manufacturer's instructions. The PCR product was purified from the amplification mixture using microCLEAN PCR clean-up kit (Microzone), and the repeat region of interest was sequenced using ABI Prism BigDye Terminator Cycle Sequencing Ready Reaction kit V.1.1. $15 \mu \mathrm{L}$ sequencing reactions comprised of 5-20 ng of DNA template, $1 \mu \mathrm{L}$ BigDye mix, $5 \mu \mathrm{L}$ Better Buffer (Microzone), 5 pmol of the forward and reverse sequencing primer (same as PCR primers), $1 \mu \mathrm{L}$ betaine and were made up with an appropriate volume of nuclease-free water. Cycling parameters for sequencing were set to $300 \mathrm{~s}$ at $95^{\circ} \mathrm{C}$, $30 \mathrm{~s}$ denaturing at $96^{\circ} \mathrm{C}, 15 \mathrm{~s}$ annealing at $55^{\circ} \mathrm{C}$, $240 \mathrm{~s}$ extension at $60^{\circ} \mathrm{C}$ and repeated for 35 cycles. Sequences were precipitated in ethanol, EDTA and sodium acetate solution and further washed in $70 \%$ ethanol. Sequences were determined on a $3730 \mathrm{xl}$ DNA Analyser capillary sequencer (Applied Biosciences) and analysed with Geneious V.6.1 sequencing software (Biomatters). 
Search of public databases and published data

We searched for published sequencing data on PFN1 in ALS, FTD and control cases from prevalently Caucasian cohorts, updated to 10 May 2013. In particular, we received from the UK10K, (http://www.uk10k.org, a large sequencing study of UK individuals with conditions not related to ALS/FTD) the allele frequency for E117G in 4732 exome sequences.

\section{Statistical methods}

Association $\mathrm{p}$ values were based on Fisher exact tests using the allele count data. Stratified population analysis was performed using the stratified Mantel-Haenszel test. All tests were performed using the R statistical software.

\section{RESULTS}

Sequence screening of PFN1 in a UK ALS cohort

Screening of the three PFN1 exons in our samples revealed no novel variant. We identified two ALS samples carrying the dinucleotide substitution leading to the previously described E117G coding change. Both patients had no ALS family history. The first patient was a male with bulbar onset ALS, age was 65 years at onset, and a very slowly progressive form of disease. The second patient was a male with limb onset ALS, age was 54 years at onset, and a disease duration of 3 years. Both patients were negative for screening of C9orf72 expansions and mutations in TARDBP, FUS and VCP.

\section{Meta-analysis of PFN1 E117G variant}

To estimate the frequency of the E117G alleles in controls, we combined several publicly available cohorts of exome-sequenced individuals, whose conditions do not overlap with the ALS-FTD spectrum. The largest control cohorts are: NHLBI Exome-sequencing dataset (4300 European-American samples), and the UK10K dataset (4732 UK samples).

We then collected all the published and publicly available data on the E117G PFN1 variant in ALS and FTD. Combining all cohorts, we obtained information on 5118 cases and 13089 controls of prevalently European descent.

We initially performed our statistical analysis using the original discovery set from Wu et al, ${ }^{4}$ with the following modifications to the control group: (A) we excluded the $1 \mathrm{~K}$ Genome Project data due to low coverage and potential shortfall in detecting rare variants; (B) we excluded the African-American samples from the Exome Variant Server, since the E117G mutation appears to be specific to European-descent populations; (C) we updated the European-descent samples as on June 2013. This analysis shows an $\mathrm{OR}=3.71$ (Fisher exact test $\mathrm{p}=0.098$ ) (table 1).

We then analysed all available datasets which included cases and controls (total of 4024 cases and 8777 controls) and took into account population stratification by using a MantelHaenszel test. This analysis showed a significant difference between groups $(p=0.036)$ and an OR of $2.22(1.1$ to $\infty 95 \%$ one-tailed CI) (table 1).

By combining all available data, also from non-case-control studies, the variant was present in 13 out of 5118 cases and 15 out of 13089 control samples conferring similar results to the Mantel-Haenszel test, and showing an OR=2.22 (95\% CI 1.1 to $\infty$ Fisher exact test $\mathrm{p}=0.036$ ) (table 1 ).

\section{DISCUSSION}

PFN1 mutations have been reported to be a rare cause of familial ALS. ${ }^{4}$ The PFN1 E117G variant has been also found in sporadic ALS patients, but its presence in numerous control individuals has raised concerns about its role in disease. ${ }^{4-11}$ With the recent advances in DNA sequencing technologies, information on one patient's whole exome or multiple candidate genes is quickly obtainable-in this context, the interpretation of variants, such as PFN1 E117G, becomes an important clinicalgenetics challenge.

In a new screen of 383 UK patients with ALS, we found two E117G carriers. No novel variant was found. In order to gain insight as to how to interpret the role of this variant, we

Table 1 Frequency of PFNI E117G variant

\begin{tabular}{|c|c|c|c|c|c|c|c|c|c|c|c|}
\hline Population & ALS & E117G & FTD & E117G & $\begin{array}{l}\text { TOTAL } \\
\text { cases }\end{array}$ & E117G & CONTROL & E117G & p Value & $\begin{array}{l}\text { OR (one-tailed } \\
95 \% \mathrm{CI} \text { ) }\end{array}$ & Reference \\
\hline \multicolumn{12}{|c|}{ Original discovery set (after exclusion of African samples in NHLBI dataset, and removal of 1000 genomes cohort) } \\
\hline North American* & 1090 & 3 & & & 1090 & 3 & 1089 & 1 & & & 4 \\
\hline European American & & & & & & & 4300 & 3 & & & $\begin{array}{l}\text { NHLBI exome } \\
\text { sequencing project }\end{array}$ \\
\hline Total & & & & & 1090 & 3 & 5389 & 4 & 0.098 & 3.71 & \\
\hline \multicolumn{12}{|l|}{ Validation datasets } \\
\hline $\mathrm{UK}^{*}$ & 383 & 2 & & & 383 & 2 & 4732 & 8 & & & $\begin{array}{l}\text { UK10K (http://www. } \\
\text { uk10k.org) }\end{array}$ \\
\hline US/Nordic/German* & 672 & 1 & 16 & 0 & 688 & 1 & 972 & 0 & & & 7 \\
\hline French & 46 & 0 & 119 & 0 & 165 & 0 & & & & & 8 \\
\hline Italian* & 1168 & 1 & 203 & 0 & 1371 & 1 & 1132 & 0 & & & 9 \\
\hline Canadian & 94 & 0 & & & 94 & 0 & & & & & 5 \\
\hline Australian & 825 & 3 & & & 825 & 3 & & & & & 11 \\
\hline Belgian* & 174 & 0 & 328 & 3 & 502 & 3 & 864 & 3 & & & 6 \\
\hline \multicolumn{12}{|l|}{ Combined analysis } \\
\hline $\begin{array}{l}\text { Total (all samples, not } \\
\text { stratified) }\end{array}$ & 4452 & 10 & 666 & 3 & 5118 & 13 & 13089 & 15 & 0.036 & $2.22(1.1$ to $\infty)$ & \\
\hline $\begin{array}{l}\text { Total }^{*} \text { (stratified Mantel- } \\
\text { Haenszel test) }\end{array}$ & & & & & 4024 & 10 & 8777 & 12 & 0.038 & $2.44(1.048$ to $\infty)$ & \\
\hline
\end{tabular}


performed an analysis on all publicly available data for the E117G variant, and we find consistent evidence for the association between E117G and ALS andFTD $(p=0.036)$, with an estimated OR of 2.44 (95\% CI 1.048 to $\infty \mathrm{p}=0.038)$.

Wu et $a l^{4}$ performed functional studies on five PFN1 mutations, including the E117G variant, demonstrating an effect on protein aggregation, actin binding and axon outgrowth. The E117G variant generally showed intermediate results between the wild-type and the other mutations, reaching significant differences from the wild-type in protein aggregate formation and in increased levels in insoluble protein fractions. Axon outgrowth inhibition was observed, but did not reach statistical significance, and actin binding appeared to be normal. ${ }^{4}$ In summary, functional characterisation of the E117G variant shows lesser changes than clear PFN1 pathogenic mutations, but supports a possible role as risk factor for ALS.

The possibility of multiple mutations contributing to ALS in one individual, ${ }^{12}$ and the role of rare variants in complex diseases such as ALS and FTD, remains to be elucidated and will require very large cohorts in order to obtain sufficiently convincing results. Recently, Geschwind and collaborators have shown that a rare variant in the MAPT gene, where mutations are known to be causative for FTD, can act as a risk factor for FTD and Alzheimer's disease. ${ }^{13}$ Similarly, we here suggest that a rare variant in PFN1, a gene in which mutations are known to be causative for ALS, can act as a risk factor for disease.

\footnotetext{
Author affiliations

${ }^{1}$ MRC Centre for Neuromuscular Diseases, UCL Institute of Neurology, London, UK ${ }^{2}$ Department of Neurodegenerative Diseases, UCL Institute of Neurology, London, UK

${ }^{3}$ National Hospital for Neurology and Neurosurgery, London, UK

${ }^{4}$ Centre for Neuroscience \& Trauma, Blizard Institute, Barts and The London School of Medicine and Dentistry, Queen Mary University of London, London, UK

${ }^{5}$ Department of Molecular Neuroscience, UCL Institute of Neurology, London, UK

${ }^{6}$ Sobell Department of Motor Neuroscience and Movement Disorders, UCL Institute of Neurology, London, UK

${ }^{7}$ UCL Genetics Institute, University College London, London, UK
}

Acknowledgements We are grateful to all patients and family members who agreed to DNA donation, without which these studies would have been impossible.

Contributors PF contributed to the design and conceptualisation of the study, analysis and interpretation of the data and drafted and revised the manuscript. JC, $T C$ and $A D$ contributed to the analysis and interpretation of the data, and revising the manuscript. $\mathrm{RH}, \mathrm{AM}, \mathrm{RO}, \mathrm{KS}, \mathrm{JC}, \mathrm{MS}$ and $\mathrm{CL}$ contributed to the analysis of the data and revising the manuscript. JH and EMCF contributed to the analysis and interpretation of the data, and revising the manuscript. VP contributed to the design and conceptualisation of the study, the statistical analysis and interpretation of the data and revised the manuscript.

Funding PF is funded by MRC/MNDA Lady Edith Wolfson Fellowship. EMCF is funded by the UK Motor Neuron Disease Association. PF and EMCF are funded by the UK Medical Research Council and the Thierry Latran Foundation.

Ethics approval Ethics approval for this project was obtained by the local University College Hospital Ethics committee.

Provenance and peer review Not commissioned; externally peer reviewed.

Open Access This is an Open Access article distributed in accordance with the terms of the Creative Commons Attribution (CC BY 3.0) license, which permits others to distribute, remix, adapt and build upon this work, for commercial use, provided the original work is properly cited. See: http://creativecommons.org/licenses/ by/3.0/

\section{REFERENCES}

1 Haverkamp LJ, Appel V, Appel SH. Natural history of amyotrophic lateral sclerosis in a database population. Validation of a scoring system and a model for survival prediction. Brain 1995;118(Pt 3):707-19.

2 Andersen PM, Al-Chalabi A. Clinical genetics of amyotrophic lateral sclerosis: what do we really know? Nat Rev Neurol 2011;7:603-15.

3 Lillo P, Hodges JR. Frontotemporal dementia and motor neurone disease: overlapping clinic-pathological disorders. J Clin Neurosci 2009;16:1131-5.

4 Wu C-H, Fallini C, Ticozzi N, et al. Mutations in the profilin 1 gene cause familial amyotrophic lateral sclerosis. Nature 2012;488:499-503.

5 Daoud H, Dobrzeniecka S, Camu W, et al. Mutation analysis of PFN1 in familial amyotrophic lateral sclerosis patients. Neurobiol Aging 2013;34:1311.e1-1311.e2.

6 Dillen L, Van Langenhove T, Engelborghs $S$, et al. Explorative genetic study of UBQLN2 and PFN1 in an extended Flanders-Belgian cohort of frontotemporal lobar degeneration patients. Neurobiol Aging 2013;34:1711.e1-5.

7 Ingre C, Landers JE, Rizik N, et al. A novel phosphorylation site mutation in profilin 1 revealed in a large screen of US, Nordic, and German amyotrophic lateral sclerosis/frontotemporal dementia cohorts. Neurobiol Aging 2013;34:1708.e11708.e6.

8 Lattante S, Le Ber I, Camuzat A, et al. Mutations in the PFN1 gene are not a common cause in patients with amyotrophic lateral sclerosis and frontotemporal lobar degeneration in France. Neurobiol Aging 2013;34:1709.e1-1709.e2.

9 Tiloca C, Ticozzi N, Pensato V, et al. Screening of the PFN1 gene in sporadic amyotrophic lateral sclerosis and in frontotemporal dementia. Neurobiology of Aging 2013;34:1517.e9-1517.e10.

10 Van Blitterswijk M, Baker MC, Bieniek KF, et al. Profilin-1 mutations are rare in patients with amyotrophic lateral sclerosis and frontotemporal dementia. Amyotroph Lateral Scler Frontotemporal Degener 2013;14:463-9.

11 Yang S, Fifita JA, Williams KL, et al. Mutation analysis and immunopathologica studies of PFN1 in familial and sporadic amyotrophic lateral sclerosis. Neurobiol Aging 2013;34:2235.e7-2235.e10.

12 van Blitterswijk M, van Es MA, Hennekam EAM, et al. Evidence for an oligogenic basis of amyotrophic lateral sclerosis. Hum Mol Genet 2012;21:3776-84.

13 Coppola G, Chinnathambi S, Lee JJ, et al. Evidence for a role of the rare p.A152T variant in MAPT in increasing the risk for FTD-spectrum and Alzheimer's diseases. Hum Mol Genet 2012;21:3500-12. 